\title{
Décadrages Décadrages
}

cinéma, à travers champs Cinéma, à travers champs

12 | 2008

Fredi M. Murer

\section{De l'art du portrait au documentaire ethnographique}

François Bovier

\section{(2) OpenEdition}

1 Journals

Édition électronique

URL : https://journals.openedition.org/decadrages/364

DOI : $10.4000 /$ decadrages.364

ISSN : 2297-5977

Éditeur

Association Décadrages

Édition imprimée

Date de publication : 10 avril 2008

Pagination : 21-30

ISBN : 978-2-9700582-7-4

ISSN : 2235-7823

Référence électronique

François Bovier, « De l'art du portrait au documentaire ethnographique », Décadrages [En ligne], 12 | 2008, mis en ligne le 05 février 2014, consulté le 03 avril 2022. URL : http://journals.openedition.org/ decadrages/364; DOI : https://doi.org/10.4000/decadrages.364 


\section{De l'art du portrait au documentaire}

\section{ethnographique}

\section{par François Bovier}

La critique assimile généralement les premiers films de Fredi Melchior Murer, réalisés entre 1961 et 1972, au domaine du cinéma expérimental suisse alémanique ${ }^{\mathbf{1}}$. Ils occuperaient ainsi une position de relative indépendance vis-à-vis de l'œuvre de maturité du cinéaste, culminant avec la réalisation de Höhenfeuer (1985), "chef-d'œuvre» consacré tant par la critique que par le public. Quand bien même adopterait-on cette perspective, la fonction de signal avant-coureur des premiers films de Murer ne devrait pas pour autant être minimisée: esquisses, ils indiquent une voie qui est encore en devenir - et qui prendra corps, sur un mode documentaire, à travers Wir Bergler in den Bergen sind eigentlich nicht schuld, dass wir da sind (1974) et sur un mode fictionnel, à travers Grauzone (1979). Le danger d'une approche auteuriste de l'œuvre de Murer, qui n'est pas dénuée de cohérence, tant thématique que stylistique, consiste à interpréter ses premiers pas dans le cinéma en regard de ses accomplissements ultérieurs, envisagés comme un dépassement de «bricolages $\mathbf{2}$ qui sont désormais intégrés à une narration qui se hausse à une dimension épique et universelle. On interrogera ici ce qui se joue dans ses premiers films réputés expérimentaux, en les rapportant au document ethnographique qu'il a réalisé sur les habitants des montagnes d'Uri. Dans Wir Bergler..., documentaire autofictionnel en ce sens qu'il s'agit d'un retour sur ses lieux d'origine, Murer interroge les stéréotypes de l'identité helvétique, dont l'image filmique est médiatisée par le paysage de montagne depuis les années 1920 au moins, en donnant la parole aux habitants de cette terre. Il se prête ainsi à une entreprise de cartographie de la paysannerie suisse, ce qui lui permet de dépasser l'art du portrait focalisé sur une personnalité ou sur un groupe d'amis qui était au centre de ses premiers films.

L'écart entre la pratique de Murer et le cinéma expérimental suisse doit être d'emblée souligné : dans les années 1960-1970, la scène suisse
1 Voir, pour l'approche à ce jour la plus complète des premiers films de Murer, le livre édité par Pro Helvetia en collaboration avec le réalisateur: Fredi M. Murer, Pro Helvetia, Zurich, 1980.

2 Peut-être est-ce ici le lieu de rappeler que Claude Levi-Strauss avait élevé le bricolage au rang de méthode heuristique, soulignant l'inventivité de ses règles sans cesse renouvelées en fonction des moyens du bord (La pensée sauvage, Plon, Paris, 1962). 
3 Voir HHK Schoenherr: cinéma expérimental, Pro Helvetia/L'Age d'Homme, Zurich/Lausanne, 1985. alémanique entretient des relations privilégiées avec l'école formelle autrichienne et allemande, tout particulièrement avec le mouvement Das Andere Kino, animé par Werner Nekes et Dore O. Si l'œuvre tout à fait remarquable de Schoenherr exploite systématiquement l'articulation différentielle entre les photogrammes, à travers le programme anti-illusionniste d'un cinéma brisé («das kaputte Kino») 3 , c'est en vain que l'on chercherait une telle entreprise de mise à nu de la machinerie filmique chez Murer (qui a néanmoins découvert les pratiques d'expérimentation en assistant régulièrement dans les années 1960 au festival international de Knokke-le-Zoute, en Belgique). Bien au contraire, le modèle de Murer est celui du portrait filmique, dérivé de la pratique de la photographie amateur: son cinéma s'enracine dans le film de famille, plutôt que dans la déconstruction des dispositifs de la représentation

1/ Chicorée (1966)

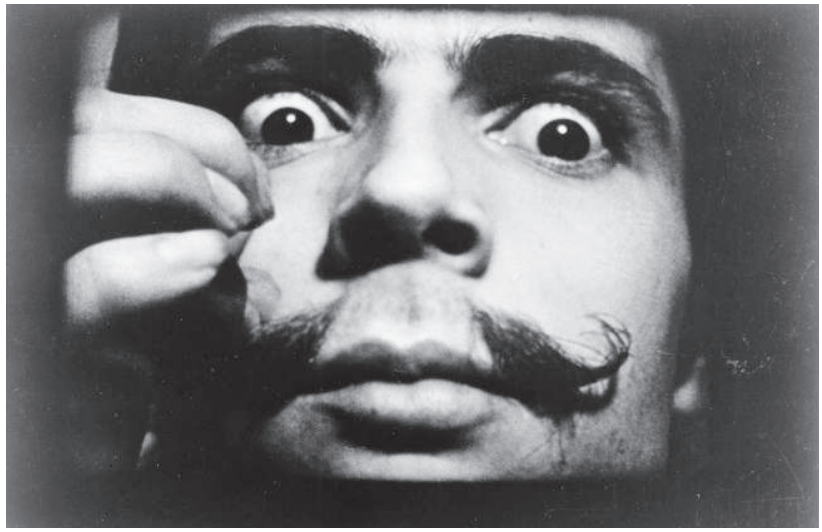

2/ Sad-Is-Fiction (1969)




cinématographique. Christopher đr Alexander, tourné en 1972-1973, corrobore l'hypothèse de l'ancrage du cinéaste dans les conventions du home movie: prototype du film de famille, ce moyen métrage porte un regard distancé et critique sur la vie de deux enfants dans leur environnement familial (à savoir le milieu d'un riche banquier, commanditaire du film). Archivant une histoire qui lui demeure impropre, Murer indexe des traces mnésiques qui s'apparentent à la structure des souvenirs reconstruits et dresse un constat sociologique sur l'éducation dans le contexte de la haute bourgeoisie.

\section{Un cinéma privé: l'archive d'une communauté d'artistes}

Les premiers portraits filmiques de Murer, s'apparentant par leurs moyens 4 à un cinema povera (comme l'on dit: arte povera), associent étroitement le modèle dépeint à la réalisation : l'entrée de Murer dans le cinéma, reposant sur une étroite collaboration entre amis, alterne entre la photographie de famille et les documents sur une figure active de la scène culturelle. En un sens, les portraits d'artistes réalisés par Murer sont indissociables de ses films centrés sur un mode de vie communautaire, tous deux reflétant et participant au Zeitgeist des années 1960, en Suisse alémanique. On assiste ainsi à un détournement généralisé du portrait filmique, avec la complicité de ses protagonistes et acteurs : Chicorée (1966) saisit sur un mode "schizo-fragmentaire» les activités du poète Urban Gwerder, tout en s'inscrivant dans un contexte de diffusion performatif, le film étant intégré aux spectacles que le poète donnait dans divers caveaux en Suisse (fig. 1); Luginbübl (1966) propose un portrait décousu et fragmentaire de l'artiste cinétique Bernard L. et de son environnement familial; Sad-Is-Fiction (1969) suit les déambulations du peintre Alex Sadkowsky, présenté comme un flâneur et un arpenteur des temps contemporains (fig. 2); Passagen (1972) restitue l'univers phantasmatique et asphyxiant de H. R. Giger, notamment en donnant à voir le peintre au travail sur des toiles où l'hybridation de l'organique et du technologique annonce l'esthétique cyberpunk (fig. 3). Dans tous les cas, il s'agit de surprendre en acte un rapport à l'œuvre, que cette dernière soit critique ou désengagée: seul le processus de création est donné à voir au spectateur, l'œuvre achevée ne constituant pas le propos de Murer.

Si l'on écarte la fiction d'anticipation politique 2069, ainsi que le documentaire fictionnel Christopher $\bullet$ Alexander et une expérimentation filmique dont la singularité ne peut être réduite (il s'agit de Vision of a Blind Man, où le cinéaste, en écho à la Lettre des aveugles de Diderot, éprouve l'expérience de la cécité et s'en remet au son dans son approche
4 Murer déclare que Pazifik (1966, près de trois heures et demie dans sa version inaugurale désormais perdue) est autofinancé à hauteur de CHF 8000.-, et qu'il a réalisé Chicorée (1966), Luginbühl (1966) et Sad-Is-Fiction (1969) grâce aux montants de primes à la qualité. En 1969, la Banque populaire suisse commandite les trois épisodes de Swissmade (dont celui, de Murer, 2069), en mettant à disposition de chaque réalisateur la somme de CHF 120 000.-. Passagen (1972) repose sur une aide de la télévision WDR et d'un investisseur privé, tandis que Christopher \& Alexander (1973) est financé par son commanditaire. A partir de Wir Bergler... (1974), Murer a recours, mais de façon encore modeste (en l'occurrence, environ CHF 300 000.-), aux financements de la Confédération et de la télévision. Voir "Neuf questions à Fredi M. Murer " [1977], dans Fredi M. Murer, op. cit., pp. 47-50. 


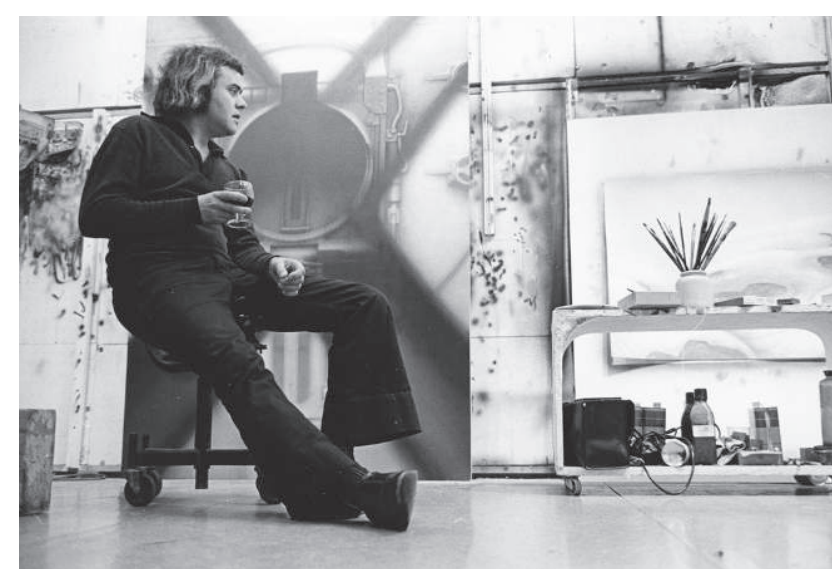

5 Balance, centré autour d'Augustin Erb, et Sylvan, autour de Sylvan Gunter, sont extraits hors du cycle intégral de Pazifik et présentés comme des œuvres à part entière. Leur homologie de structure avec les portraits d'artistes, tout particulièrement Chicorée et Luginbühl, est patente.

tactile, haptique, d'un espace dont il ne parvient pas à prendre la mesure (fig. 4)), les premiers films de Murer empruntent deux voies cinématographiques parallèles: s'inspirant de l'art du blason, ses portraits sont soit monographiques, soit communautaires. Mais il faut préciser que la distinction entre le collectif et l'individuel est arbitraire: Murer a ainsi $\mathrm{pu}$ autonomiser sous forme de courts métrages certains portraits qui entrent dans sa fresque sur la jeunesse marginale à laquelle il appartient (Pazifik - oder die Zufriedenen, 1966) 5. C'est néanmoins la voie communautaire que l'on examinera, car elle innerve le geste documentaire de Murer. Pour le dire sans détour, le cinéaste évolue d'une marginalité revendiquée à une altérité (mais tout aussi bien et indissociablement une propriété, une identité helvétique) qu'il reçoit en héritage de sa famille. Dans ses premières réalisations, il recueille les errances d'une bohème




artistique (notamment le modus vivendi d'un squat, dirait-on aujourd'hui, inventant un nouvel espace privé sur une base collective, mais dont on perçoit mal quelles sont les activités publiques et citoyennes). Dans Wir Bergler..., document rare pour ne pas dire unique en son genre en Suisse, il ausculte l'enracinement de paysans de montagne dans leur terrain. Ce double mouvement d'exterritorialité à l'espace public et d'ancrage dans une culture agraire engage des rapports de classes opposés. De plus, cette ambivalence est redoublée par le déplacement du réalisateur lui-même dans l'espace géographique, suivant une topographie duelle de l'habitat humain: Murer quitte les montagnes où il a grandi pour s'installer à Zurich, avant d'aller à Londres; retournant dans son pays natal, le cinéaste recueille une mémoire collective ${ }^{6}$ qui est authentique mais menacée de disparition.

\section{La béatification des saints : péril en la demeure}

Pazifik - oder die Zufriedenen rejoue sur un mode carnavalesque et festif les jours qui précèdent l'expulsion hors du paradis. Pazifik, c'est le nom d'une villa de Plattenstrasse à Zurich, occupée par une communauté anti-conformiste (Murer et un groupe d'amis) mais qui est vouée à la démolition. Murer en tire un film expérimental, conçu par épisodes et encadré par une fête. Sept habitants (dont le réalisateur) se mettent imaginairement en scène sous l'objectif de la caméra, à travers des masques dont l'artificialité et la théâtralité sont accusées (avec notamment les figures fantasmées du funambule (fig. 5), du peintre et de l'ermite); le registre de la mascarade, reflet déformé du théâtre social, est explicitement revendiqué. La logique de construction du film est celle de l'attraction; les liens entre les plans se font par association libre d'idées. La

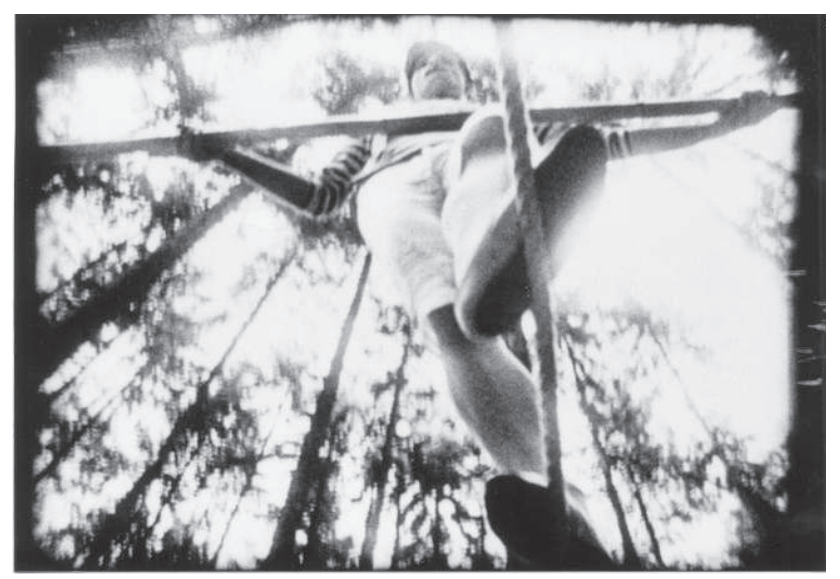

6 Murer s'intègre dans les vallées d'Uri en tant qu'enfant du pays, comme il le souligne lui-même; retournant en 1971 dans la localité uranaise d'Amsteg pour les funérailles de son père, il découvre la popularité que celui-ci avait acquis auprès de la population: "Lui-même fils d'un paysan de montagne, il avait été pour les gens de sa région une sorte d'avocat. Ils venaient le voir avec leur déclaration d'impôts ou pour lui faire écrire une lettre. Ils furent des centaines sur sa tombe. Cela éveilla ma première curiosité. Mon désir de réaliser un film sur ces montagnards dépendait peut-être aussi du fait que j'espérais en apprendre d'avantage sur mon père, que j'ai toujours connu très taciturne. Le médecin de mon père me prit alors avec lui dans ses tournées de consultations qu'il effectuait le sac à dos bourré de pastilles, de suppositoires et de kirsch. En qualité de fils de mon père et d'accompagnateur du médecin, je fus partout aussitôt accepté." (Propos rapportés par Pierre Lachat, "Vision of a Blind Man ou peut-être dois-je apprendre à crier ", dans Fredi M. Murer, op. cit., p. 28). 
7 II faut lire à travers le jeu de l'anagramme et des permutations de signifiants: "Francis Picabia Rrose Sélavy" (c'est-à-dire le nom du personnage de travesti qu'incarne Duchamp et qu'a photographié Man Ray). René Clair, sous l'instigation de Picabia, porte à l'écran cette signature, non sans la transformer en recourant au procédé de la diérèse: "Francis Picabia arrose Sélavy ". Dans Entr'acte, on voit ainsi Duchamp et Man Ray, qui jouent aux échecs sur les toits du théâtre des Champs-Elysées, se faire doucher par Picabia hors champ, à l'aide d'un tuyau d'arrosage. Sur ce point, voir Patrick de Haas, Cinéma intégral. De la peinture au cinéma dans les années 20, Transéditions, Paris, 1985. référence qui prévaut est le régime du burlesque et du music-hall, comme l'attestent telles citations détournées d'Entr'acte (René Clair, Francis Picabia, Erik Satie, France, 1924): Murer décompose à travers deux plans disséminés dans son film la scène où Picabia arrose Duchamp sur les toits d'un théâtre, celui-ci jouant aux échecs avec Man Ray (en référence à L'oil cacodylate, tableau de Francis Picabia composé de signatures d'artistes et d'écrivains, dont celle qui est évoquée ici: «en 6 qu'habilla rrose Sélavy»7). Premier élément détourné : les personnages jouent aux quilles, avant de se faire arroser par une pluie battante (économiquement réalisée en frottant le côté de l'émulsion de la pellicule avec du papier d'émeri). Second élément retourné : sur les toits d'un immeuble, un personnage (avec une moustache hitlérienne) gît mort; surgit alors un gangster armé d'un browning (déplacement de l'incipit d'Entr'acte: Picabia et Satie arment un canon et tirent à bout portant sur le public). Dans le premier portrait, l'enchaînement de deux séquences permet de faire le lien entre ces plans: lors d'une des occurrences du jeu de quilles, un serviteur entre dans le champ et tombe raide mort. La musique, lorsqu'elle intervient, a également une fonction attractionnelle: elle réinscrit le contexte de la foire et du Grand Guignol. Plusieurs motifs refont régulièrement surface dans le film, introduisant un certain ordre dans la logique du coq-à-l'âne qui constitue le seul fil conducteur auquel se tient Murer: le jeu de quille, les repas pris communautairement, mais également le rapport à la création artistique, les jeux de travestissement ou encore le motif du pistolet ordonnent par leur réitération ce bric-àbrac (Murer ne suivant aucun scénario, ni plan préétabli).

Les liaisons locales entre plans, on l'a dit, reposent sur les mécanismes de la condensation et du déplacement, sur les figures de la métonymie et de la métaphore, qui sont au centre des réflexions de Freud sur le travail du rêve. Après avoir introduit le motif de la mort (un soldat porte un masque à gaz; dans le plan suivant, il est décédé, étant démasqué), le portrait se concentre sur la figure d'un peintre, en l'occurrence Daniel Bamert. Suite à une représentation de la Maya de Goya, l'artiste apparaît mort, avant de se relever, portant des lunettes organiques et contreproductives, les verres étant remplacés par des feuilles. Il peint alors une toile; une femme nue (renvoi évident à la Maya) apparaît sous les traits de son pinceau, malgré sa cécité passagère (la fonction des lunettes étant inversée). Le plan suivant est centré autour d'une pomme, métonymie du pêché originel; s'ensuit une scène de repas, où sont consommées des cuisses de poulets. Le principe de composition de la séquence est aisément identifiable: un motif en appelle un autre, les analogies permettant d'opérer une série de déplacements. 
Au sein même des plans, on assiste à un procédé analogue d'assemblage d'indices renvoyant à des fonctions sociales déterminées, mais dont l'ordonnancement est régulièrement contrecarré par l'aboutage d'éléments hétéroclites. Aussi Murer peut-il assembler au sein du même plan une seringue, un hameçon, une loupe et une boule à thé: l'élément piquant permet de relier la seringue (fonction médicale, monde de la drogue) et l'hameçon (fonction des loisirs); l'élément ovale permet d'associer la loupe (fonction du détective) à la boule à thé (monde de la bonne société). Finalement, c'est l'hameçon qui devient l'élément dominant dans le processus associatif qui suit: le plan suivant cadre un vers de terre, appât pour que le poisson morde; suit alors un plan tourné dans un musée d'histoire naturelle, où sont exposés des squelettes de dinosaure. Le modèle, en ce cas, est à chercher du côté de la postérité du dadaïsme, Breton s'appropriant dans le premier manifeste du surréalisme la définition de l'image poétique par Reverdy - "plus les rapports des deux réalités rapprochées seront lointains et justes, plus l'image sera forte $\boldsymbol{8}$ - tandis que les montages verbaux incongrus de Lautréamont - «beau comme la rencontre fortuite sur une table de dissection d'un parapluie et d'une machine à coudre » $\mathbf{9}$ - font figure de modèle pour les surréalistes.

Portrait d'amis, dialogue entre pairs, écriture semi-automatique, exaltation de la création et de la liberté individuelle, sans impliquer d'engagement politique (question d'époque et de lieu, probablement), voilà quelques traits récurrents des premiers portraits filmiques de Murer. Leur spontanéité est indéniable, entretenant une confusion décomplexée entre le cinéma amateur, le genre documentaire et le style "art et essai». Wir Bergler... ne constitue pas un film de transition, un passage à une maîtrise de la forme et du discours cinématographiques, qui s'actualiserait par la suite sur un mode fictionnel. Bien au contraire, on assiste là à un dépassement, à un accomplissement (Aufhebung) des premières réalisations qui se font encore, malgré l'éventuelle noirceur du propos, sur un mode enjoué et amateur. Murer atteint à une radicalité en acte, arc-boutée à une expérience et à une mémoire concrètes, telluriques: Wir Bergler... participe, dans le contexte suisse alémanique, au vaste mouvement de renouvellement du cinéma ethnographique et anthropologique tel qu'il se pratique dans divers pays (que la critique a caractérisé, épinglé, sous les étiquettes de : cinéma direct, cinéma vérité, cinéma du vécu, Candid Eye Cinema). C’est un film qui rejoint la théorie du théâtre épique et didactique de Brecht, mais qui fait porter l'accent sur l'exposition d'une situation plutôt que sur l'effet d'étrangeté (Verfremdung-Effect) : en donnant la parole aux protagonistes du drame,
8 Breton cite la définition de l'image poétique articulée par Pierre Reverdy dans la revue NordSud, en mars 1918 (André Breton, Manifestes du surréalisme [extrait du premier manifeste, publié en 1924], Gallimard, Paris, 1963, p. 31).

9 Man Ray a ainsi pu intituler l'une de ses photographies, en hommage à Lautréamont, L'énigme d'Isidore Ducasse (1920), qui est reproduite en page de couverture du numéro inaugural de $L a$ révolution surréaliste en 1924. Voir le Comte de Lautréamont [Isodore Ducasse], Les Chants de Maldoror, chant VI, 1 (Jean-Luc Steinmetz, éd.), Flammarion, Paris, 1990 [1869], p. 289 ("[Mervyn] est beau comme la rétractilité des serres des oiseaux rapaces; ou encore, comme l'incertitude des mouvements musculaires dans les plaies des parties molles de la région cervicale postérieure; ou plutôt, comme ce piège à rats perpétuel, toujours retendu par l'animal pris, qui peut prendre seul des rongeurs indéfiniment, et fonctionner même caché sous la paille; et surtout, comme la rencontre fortuite sur une table de dissection d'une machine à coudre et d'un parapluie."). 
10 Le découpage est tributaire des lieux du tournage. Des cartons, comprenant des déclarations d'indigènes, annoncent le début de chaque partie: "1. Vallée de Göschenen: "ll faut qu'il y ait un changement tout différent, dans tout ça.'" "2. Vallée de Schächen: "Ces enfants ont déjà hérité de la passion de la montagne...." "3. Vallée de Maderan: 'Mais parfois il nous semble ici que nous sommes en quelque sorte des citoyens de deuxième catégorie. '". aux agents de leur propre vie, Murer réalise un film politique avec et pour la paysannerie de montagne, dont il est issu.

\section{Autofiction et anthropologie sauvage: "Mais parfois il nous semble ici que nous sommes en quelque sorte des citoyens de deuxième catégorie "}

Découpé en trois mouvements $\mathbf{1 0}$, comme une partition musicale, Wir Bergler... chronique la rude vie des habitants de vallées alpestres retirées, à travers le mode du passé antérieur (les gestes, le travail, le rapport à la terre des montagnards, l'étirement de la temporalité qui est scandée par le labeur à accomplir à heures fixes et inlassablement, tout concourt à nous plonger dans un temps archaïque, difficilement assignable). La première séquence, à caractère allégorique, situe le degré d'éloignement de ces montagnes: la caméra, placée dans une voiture, capte le défilement des tunnels à travers la bretelle d'autoroute du Gothard; trace de l'activité humaine, ces montagnes trouées signifient que l'on se dirige vers un ailleurs dont l'accès est lointain. Sur place, Murer s'affronte à l'image stéréotypée du paysage suisse, à son caractère de carte postale : comment éviter l'écueil de la belle image, du chromo et du pittoresque? Murer y parvient à travers deux stratégies croisées. D’une part, sa caméra porte un regard analytique sur le milieu filmé : c'est le rapport des paysans à la terre qui est ausculté, à travers un regard qui s'apparente parfois à celui de l'entomologiste. D'autre part, Murer se prête à l'art d'un autoportrait déplacé : c'est son histoire, son passé qu'il filme. A cet égard, l'utilisation des archives et le rapport aux sources (car c'est bien d'un retour aux sources dont il s'agit) sont exemplaires : Murer, pendant la première demi-heure du film, confronte l'image des lieux à des photographies du début du siècle et à des archives filmiques; par la suite, il s'affranchit de cette médiatisation, restituant directement l'être-là (Dasein) de la terre et de son habitation. S'il compare deux images dans la première partie de son film, l'une présente et l'autre irrémédiablement passée, Murer confronte également les paysans de montagne à leur propre histoire, ou plutôt au récit qu'ils lui en ont fait: il filme les habitants qui écoutent leurs propres paroles, celles-ci étant également restituées sur la bande son; le spectateur est ainsi placé à distance de ces tranches de vies, dans une position d'objectivité quasi scientifique.

L'image du paysage de montagne, constitutive de la construction identitaire du cinéma suisse, est ici redonnée dans sa matérialité : à travers un cadrage d'une rare rigueur et précision, dont la dimension plastique est indéniable, Murer souligne l'enracinement des paysans à la terre (très concrètement, comme lorsqu'il s'agit de débarrasser un terrain de ses pierres) et leur relation au bétail (ainsi qu'à la production qu'ils 


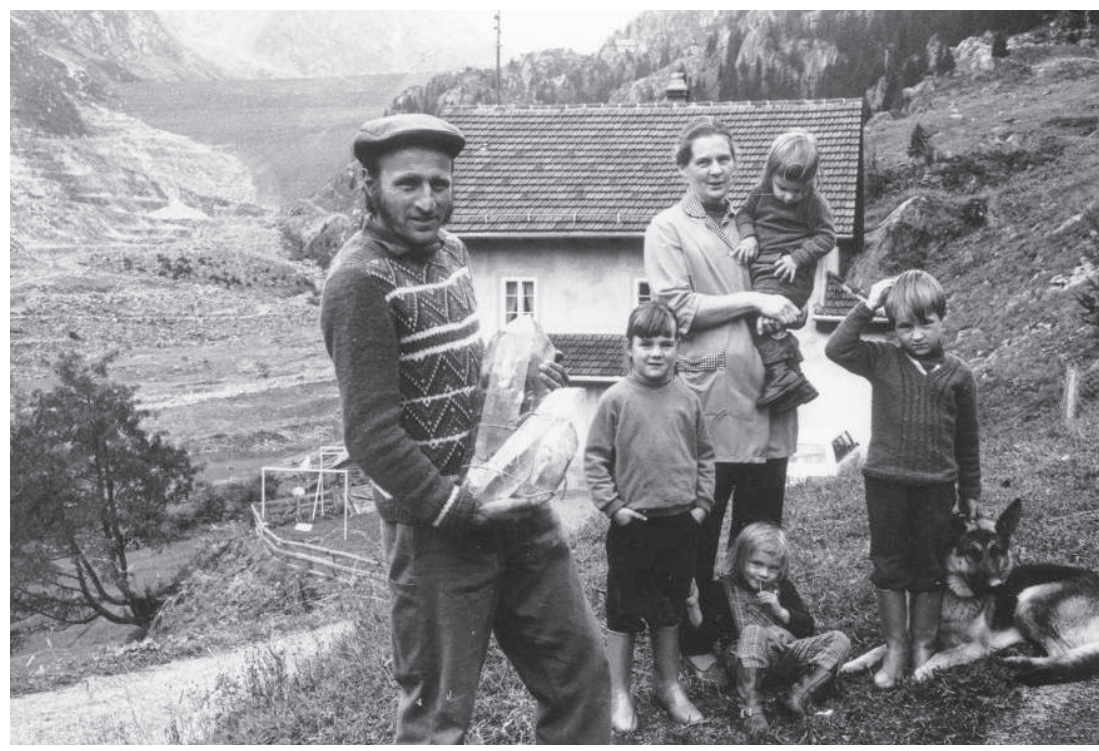

en tirent), sans ne rien masquer de la dureté de leurs conditions de vie. Chronique d'un autre temps, documentaire anthropologique sur un mode de vie ancestral, Wir Bergler... déconstruit le stéréotype du film de montagne, donnant à percevoir au spectateur le prototype même de la vie alpestre. Il s'agit pour Murer de composer un portrait de groupe, de documenter les activités d'une communauté. Pour ce faire, le cinéaste a recours au portrait posé, oscillant entre le tableau vivant et l'album de photo de famille (fig. 6). Le décalage entre la spontanéité des paroles, le recours à l'histoire orale, et la conventionalité dans la construction de l'image (non seulement la pose que prennent les villageois, mais encore la découpe qu'opère le cadre), est encore accentué par le recours à la postsynchronisation du son (qui autorise un jeu d'écoute de son propre discours). Mémoire collective, archives d'une collectivité, Wir Bergler... atteint à la "puissance de la fabulation" que Deleuze décelait chez Perrault $\mathbf{1 1}$ et qui revient somme toute à donner la parole à un peuple qui constitue une minorité. C'est en ce sens que le documentaire de Murer acquiert une valeur ethnographique, bien plus encore que par sa restitution de rites et de coutumes, de processions et autres cérémonies folkloriques. Ceux-ci scandent la vie quotidienne des paysans de montagne, tout comme la culture de la terre et le travail à la ferme ponctuent la journée. Les activités civiques sont également présentées, comme à travers cette séquence qui documente une assemblée de la Landsgemeinde, les habitants votant à main levée, ou encore à travers la captation de la
6/ Wir Bergler in den Bergen sind eigentlich nicht schuld, dass wir da sind (1974)

11 "Quand Perrault s'adresse à ses personnages réels du Québec, ce n'est pas seulement pour éliminer la fiction, mais pour la libérer du modèle de vérité qui la pénètre, et retrouver au contraire la pure et simple fonction de fabulation qui s'oppose à ce modèle. Ce qui s'oppose à la fiction, ce n'est pas le réel, ce n'est pas la vérité qui est toujours celle des maîtres ou des colonisateurs, c'est la fonction fabulatrice des pauvres, en tant qu'elle donne au faux la puissance qui en fait une mémoire, une légende, un monstre. [...] Ce que le cinéma doit saisir, ce n'est pas l'identité d'un personnage, réel ou fictif, à travers ses aspects objectifs et subjectifs. C'est le devenir du personnage réel quand il se met lui-même à ,fictionner, quand il entre sen flagrant délit de légender, et contribue ainsi à l'invention de son peuple." (Gilles Deleuze, L'image-temps, Minuit, Paris, 1985, p. 196). 
12 Louis Zukofsky, dans son long poème épique " $A$ ", écrivait: "Un objectif - rayons de l'objet ramenés à un foyer, / Un objectif - la nature comme créateur - / Désir de ce qui est objectivement parfait, / Inextricablement l'orientation des singularités / historiques et contemporaines." A partir de ce credo qui s'origine dans l'écriture poétique, Zukofsky fixe dans un manifeste les règles auxquelles doit se tenir une écriture authentique: "Un poème comme objet. [...] Ou la nature comme créateur, parfait existant, expérience parfaisant l'activité de l'existence, la faisant - théologiquement, peutêtre - comme l'Ineffable. [...] Le contexte - Le contexte ayant forcément affaire à un monde extérieur - Le désir de ce qui est objectivement parfait, inextricablement l'orientation des singularités historiques et contemporaines - Désir de tout placer - tout adéquatement, parfaitement, partie intégrante d'un contexte, faisant un avec lui." (Louis Zukosfksy, "Un objectif", Un Objectif et deux autres essais, Ed. Royaumont, Châtillon-sous-Bagneux, 1989, traduit par Pierre Alféri, p. 14 et p. 17). réunion du conseil du village en un panoramique à 360 degrés. La chronique de Murer porte sur de larges cycles, tels que la montée aux alpages et la désalpe, découpant l'espace et rythmant les saisons. En un sens, la temporalité que met en scène Murer est achronique, dénuée de toute trace de la modernité et de tout outil industriel, plusieurs générations coexistant dans le même habitat.

En dernière analyse, on peut soutenir que la force de Wir Berg$l e r .$. repose sur un mode d'énonciation objectivant, Murer trouvant une distance appropriée à son sujet. Il réactualise ainsi sur un mode filmique le programme que les poètes objectivistes avaient établi dans les années 1930, c'est-à-dire une écriture qui repose sur la "sincérité», la «singularité» et l'«objectification»12. Par cette comparaison, l'on mesure bien l'étendue de la distance parcourue entre Pazifik et Wir Bergler... : la fascination narcissique envers la figure de l'artiste s'efface pour faire place à la «nature comme créateur» et les «singularités historiques et contemporaines» qui la traversent et la travaillent. 